\title{
Medikale Landschaften. \\ Das Sanatorium als gedachte und gelebte Gesundheitsgeographie
}

EBERHARD WOLFF

Das Untersuchen medikaler Räume ist keine Erfindung des jüngsten "spatial turn". Die historische Geographie von Krankheiten, um nur ein Beispiel zu nennen, stellt einen Teilbereich der traditionellen Medizingeschichtsforschung dar (vgl. Ackerknecht 1963). Im Zusammenhang mit der neueren geistes- und sozialwissenschaftlichen Forschung wurden "Raum" und "Gesundheit" vor allem im angloamerikanischen Bereich seit den 1990er Jahren unter dem Begriff der "Therapeutic Landscapes“ zusammengebracht (Gesler 1992). Unter diesem Terminus hat sich eine bunte Mischung unterschiedlichster Ansätze aus dem breiten Feld der "Medical Anthropology" zusammengefunden (z.B. Williams 2007a; Gesler 2005; Williams 1999). Der originäre Ansatz war indes eher restriktiv. Es ging zunächst vornehmlich um das Entdecken gesundheitsförderlicher Orte. Mittlerweile sind die Ansätze wesentlich breiter. In den Fokus gerieten etwa auch Wartezimmer oder Yoga-Zentren als "Therapeutic Landscapes“. Nicht ganz zu Unrecht wurde das Forschungsfeld bereits als anekdotisch kritisiert (Williams 2007b: 9). Was es m. E. vor allem vermissen lässt, ist eine Auseinandersetzung mit dem von Williams verwendeten Begriff der "Landschaft". Dabei dürfte gerade dieser Begriff für die kulturwissenschaftliche Forschung einiges Potential besitzen. Und mehr noch: In der Forschung zu den „Therapeutic Landscapes" gibt es zwar viele Einzelanalysen solcher therapeuti- 
scher Landschaften, aber selten exemplifizieren sie explizit, wie ein bestimmtes Konzept von Landschaft als Instrumentarium für konkrete kulturwissenschaftliche Analysen eingesetzt werden kann, um die häufig zu beklagende Diskrepanz zwischen der Theorie und ihrer empirischen Umsetzung etwas zu überbrücken. Der vorliegende Beitrag versucht, diese Lücke ein Stück weit zu schließen.

Im Zusammenhang mit der derzeitigen immensen Konjunktur der Untersuchung von sozialen und kulturellen Phänomenen unter dem Aspekt des Raums ist auch der Begriff der "Landschaft" mehr in den Vordergrund getreten (vgl. Kaufmann 2005). Nicht zuletzt die Volkskunde/Kulturwissenschaft unternimmt neue Ansätze, Landschaft als „kulturwissenschaftliche Kategorie“ zu verwenden (Seifert/Krebs 2010; Fischer 2008). ${ }^{1}$ Einer der innovativeren Aspekte dabei ist, dass sich das Konzept der "Landschaft" immer mehr vom zwangsweisen Zusammenhang mit dem Naturraum löst. „Landschaften" müssen in dieser Sicht nicht notwendigerweise auch Naturräume (im Sinne etwa von "die Landschaft") sein. "Stadtlandschaften" etwa können genauso als kulturwissenschaftliche Landschaften betrachtet werden, die grundsätzlich nichts mehr mit einem Naturraum zu tun haben müssen. Noch deutlicher wird die Abstrahierung des Landschaftsbegriffs in Termini wie "Medienlandschaft" oder "Bildungslandschaft", die mehr als nur metaphorisch verstanden werden, sich damit letztlich vom konkreten Ortsbegriff lösen und von virtuellen Räumen bzw. ebensolchen Landschaften ausgehen. Als Folge dieser Ausweitung setzt sich die schon länger bekannte Einsicht nun konsequenter durch, dass "Landschaft" nicht gegeben, sondern im Grunde einerseits produziert ist, etwa durch soziale Strukturen, andererseits aber auch subjektiv konstruiert ist, etwa durch kulturelle Wahrnehmungsformen (Löw 2001, exemplarisch Kaufmann 2005). In diesem Sinne spricht der amerikanische Kulturgeograph und Landschaftstheoretiker Denis Cosgrove sinngemäß und erfrischend abstrakt von Landschaft als an verschiedenen Orten und $\mathrm{zu}$ verschiedenen Zeiten unterschiedlich konzeptualisierter, konstruierter, erfahrener und symbolisierter Umwelt (nach Hoey 2007: 300). Selbst eine Naturlandschaft wird in diesem Sinne erst

1 Siehe auch den Arbeitskreis Landschaftstheorie (www.landschaftstheorie.de). 
durch Wahrnehmungsformen und Aufladung mit einer Bedeutung, etwa durch Touristen oder Tourismus-Marketing, zu einer solchen kulturellen „Landschaft". Landschaft ist in diesem Sinne, so eine weitere definitorische Annäherung, ein Symbol, das von einem Subjekt hergestellt oder verwendet wird, um damit Sinn in einem Raum zu vergegenwärtigen. ${ }^{2}$ Das Konzept "Landschaft" lässt sich damit für jegliche Ausformung von Raum anwenden. Es spiegelt in besonderer Weise die Perspektive, in der Räume heute geisteswissenschaftlich untersucht werden.

\section{Das Sanatorium als medikale Landschaft}

Dieser Beitrag möchte sich dem analytischen Potential nähern, das ein solches Verständnis von Landschaft für die Forschung im Bereich der Medikalkultur haben könnte und versucht, "Landschaft" als Instrument des Verstehens einzusetzen. Er untersucht mit einem einzelnen Sanatorium das historische Beispiel einer medikalen „Mikrolandschaft" und fragt, welche Vorstellungen von gesunder Lebensweise sich in dieser Landschaftskonstellation spiegeln. Der Beitrag wendet verschiedene analytische Perspektiven an, die nicht nur als Instrumentarium zur Untersuchung dieser speziellen medikalen Landschaft dienen, sondern auch Vorschläge für die Untersuchung anderer medikaler Landschaften machen sollen.

Bei dem hier untersuchten Beispiel handelt es sich um das 1904 in Zürich eröffnete Sanatorium „Lebendige Kraft" des Arztes, Naturheil-Anhängers, Ernährungsreformers und RohkostPropagandisten Max Bircher-Benner, auf den auch das international bekannte „Birchermüesli“ zurückzuführen ist (Abb. 1) (Wolff 2010a, Wolff 2010b, dort weiterführende Literatur). Nach der Gründung entwickelte sich das Sanatorium schnell zu einem "Rohkost"-Mekka mit internationalem Renommee. In der Einrichtung mussten die Patientinnen und Patienten nach den Vorstellungen von Bircher-Benners „Ordnungstherapie“ leben, die neben vegetarischem Essen, häufig Rohkost, auch viel Umgang in frischer Luft, Wasseranwendungen etc. vorsah. "Genussgifte" wie

2 In Anlehnung an eine Definition in www.wikipedia.de, Artikel „Landschaft“, Zugriff Oktober 2009). 
Tabak, Kaffee oder Zigaretten waren geächtet. Der Tagesablauf war nach einem strengen, beinahe zwinglianischen Ordnungssystem strukturiert mit frühem Aufstehen, einem Morgenspaziergang noch vor dem Frühstück und einer frühen Bettruhe.

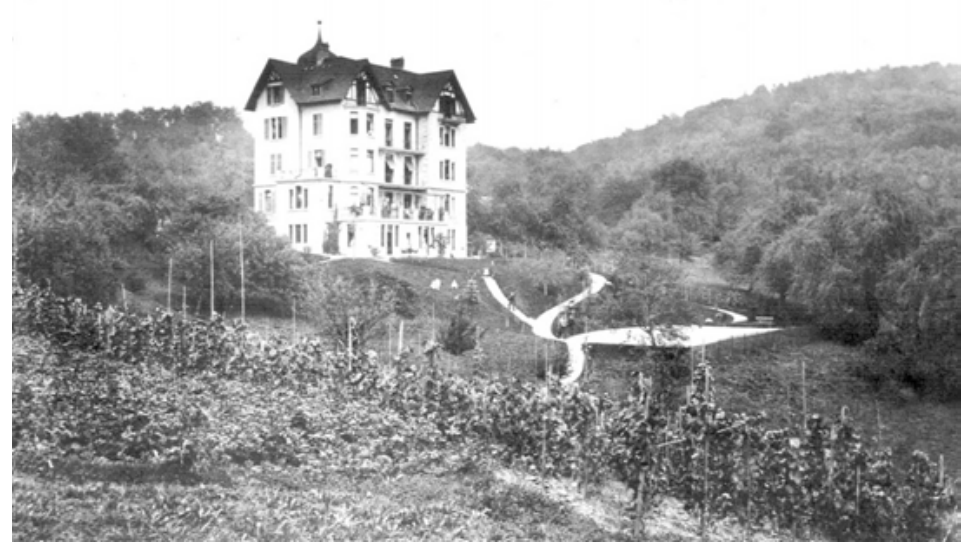

Abbildung 1: Das Sanatorium "Lebendige Kraft" auf dem Zürichberg im Zustand des Gründungsjahrs 1904 (Bircher-Benner-Archiv, Universität Zürich).

Wodurch aber wurde das Sanatorium zu mehr als einer naturheilkundlichen Kureinrichtung, nämlich zu einer medikalen Mikrolandschaft im skizzierten Sinne? Allgemeiner formuliert: Was macht einen (medikalen) Raum zur "Landschaft" im abstrakten kulturwissenschaftlichen Sinn? Im Folgenden sollen in mehreren Schritten unterschiedliche Perspektiven an dieses Beispiel angelegt werden, um das Sanatorium als eine medikale Landschaft zu analysieren. Es geht dabei nicht um eine erschöpfende Untersuchung dieses Beispiels, dieses dient vielmehr als exemplarischer Raum zur Herausstellung charakteristischer Aspekte dieser kulturellen Landschaft, um abstraktere Merkmale von kulturellen Landschaften herauszuarbeiten. Dabei wird zusätzlich eine Reihe von weiteren raumbezogenen Begriffen wie z.B. "Ziel“ oder "Lage" bewusst herangezogen (und kursiv hervorgehoben), da auch 
sie helfen, den Landschaftscharakter des Sanatoriums deutlicher hervortreten $\mathrm{zu}$ lassen.

\section{Das Sanatorium als Ort}

Um zu einer „medikalen Landschaft“ zu werden, musste das Sanatorium zunächst ein definierter konkreter Ort (im Sinne eines Raums zunächst ohne Binnendifferenzierung) werden. Das klingt wie eine Selbstverständlichkeit, war aber ein komplexerer Vorgang. $\mathrm{Zu}$ ihm gehörte unter anderem der Neubau des Sanatoriumsgebäudes. Das Sanatorium setzte sich architektonisch zunächst allerdings nur teilweise von der Umgebung ab. Der Heimatstil des Hauptgebäudes war zur Gründungszeit in diesem Quartier verbreitet. Zum Ort wurde das Sanatorium etwa durch die Herrichtung des ihn umgebenen Grundstücks, aber auch durch die Benennung mit einem speziellen Namen, der sich durch Anzeigen und Prospekte gedanklich festsetzen konnte. Auf Karten und Plänen wurde dieser Name räumlich festgelegt.

Indem an diesem Ort eine ganz spezifische Therapiemethode durchgeführt wurde mit einer Lebensweise, die sich vom mehrheitlichen Alltagsverhalten der Umgebung unterschied, wurde das Sanatorium zu einem spezifischen Ort etwa als vegetarischer oder "Rohkost"-Raum, der sich von der Umgebung in dieser Hinsicht unterschied, etwa von den Restaurants der Umgebung, in denen Fleisch und Alkohol serviert wurde. Gerade ein solches Erscheinungsbild als Insel (ähnlich Hoyez 2007) einer bestimmten Lebensweise half mit, seinen Charakter als spezifischer Ort zu stärken. $\mathrm{Zu}$ einem Ort wurde das Sanatorium auch dadurch, dass es zunehmend zum (ersehnten oder realisierten) Ziel von Anhängern der Ordnungstherapie wurde, die dort wiederum eine Art "Community“ bildeten. Der Ort war damit gleichzeitig Treffpunkt. 


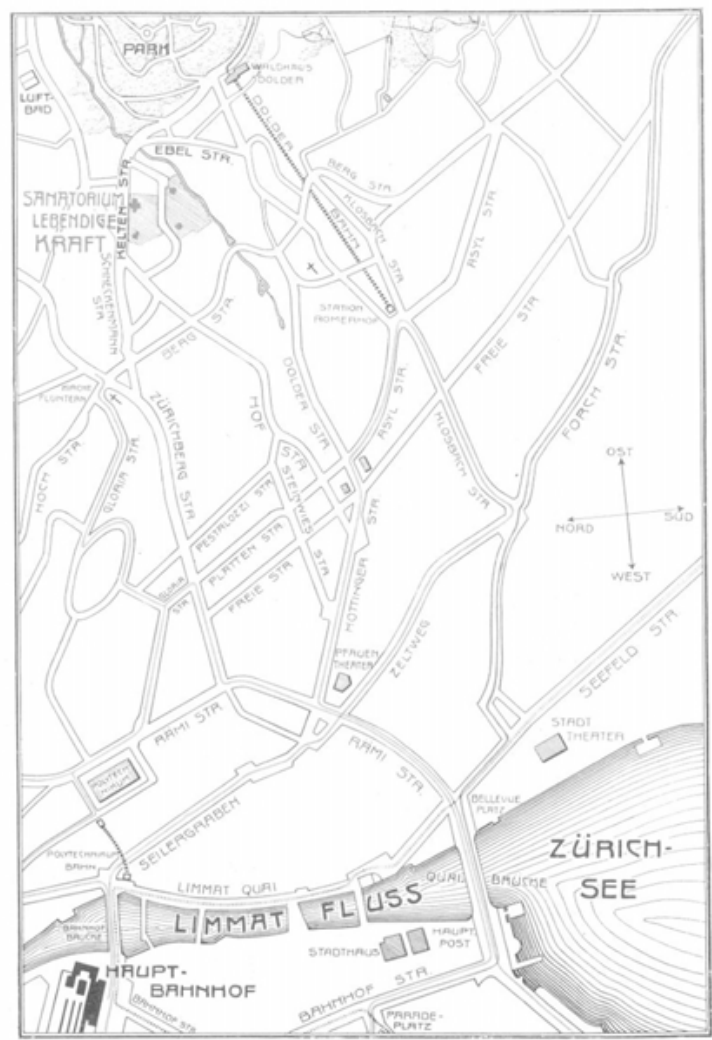

Situationsplan mit den Linien der elektrischen Strassenbahn.

Abbildung 2: Lageplan des Sanatoriums mit eingezeichneten

Straßenbahnlinien aus einem Sanatoriumsprospekt von 1907. (BircherBenner-Archiv, Universität Zürich).

\section{Landschaft als Verdichtungszone einschlägiger Merkmale}

Eine kulturelle Landschaft konstituiert sich, das ist zunächst ebenso selbstverständlich, durch spezifische Merkmale, die dort in gehäufter Form auftreten. Um zu einer „medikalen Landschaft“ zu werden, bedurfte das Sanatorium einer ebensolchen Verdichtung 
spezifischer Merkmale. Erkennbar waren diese Merkmale z.B. an einer Ansammlung sichtbarer gesundheitsrelevanter Orte auf dem Sanatoriumsgelände, innerhalb wie außerhalb der Gebäude. Besonders auffällig waren die letzteren. Das gesamte Gelände war eine konstruierte Naturlandschaft, nicht zuletzt mit den Lufthütten für regelmäßige Luftbäder. (Abb. 3). Eine wichtige Rolle spielten die Spazierwege, die nicht allein für die Frischlufttherapie angelegt wurden, sondern auch Leitbahnen für die bewusste Rezeption der inszenierten Natur darstellten. Selbst die auf dem Gelände angelegten Gemüsebeete hatten mehr als die praktische Funktion, Rohstoffe für die Rohkost-Küche zu liefern. Sie waren ebenso öffentliche symbolische Repräsentationen des Rohkostgedankens wie auch der Bewegung im Freien, wenn Patienten in ihnen arbeiteten. Auch das Innere der Gebäude war eine Ansammlung gesundheitsrelevanter Orte, seien es der Vortragssaal oder die Bibliothek für die intellektuelle Vermittlung der Ordnungstherapie oder die Räume für Wasser-, Licht- oder elektrotherapeutische Anwendungen (Abb. 4). Eine zentrale Funktion als Ort der Gesundheit hatte der Speisesaal (Abb. 5), in dem der Kern der Ordnungstherapie, die vegetarische bzw. Rohkost-Ernährung, praktiziert wurde.

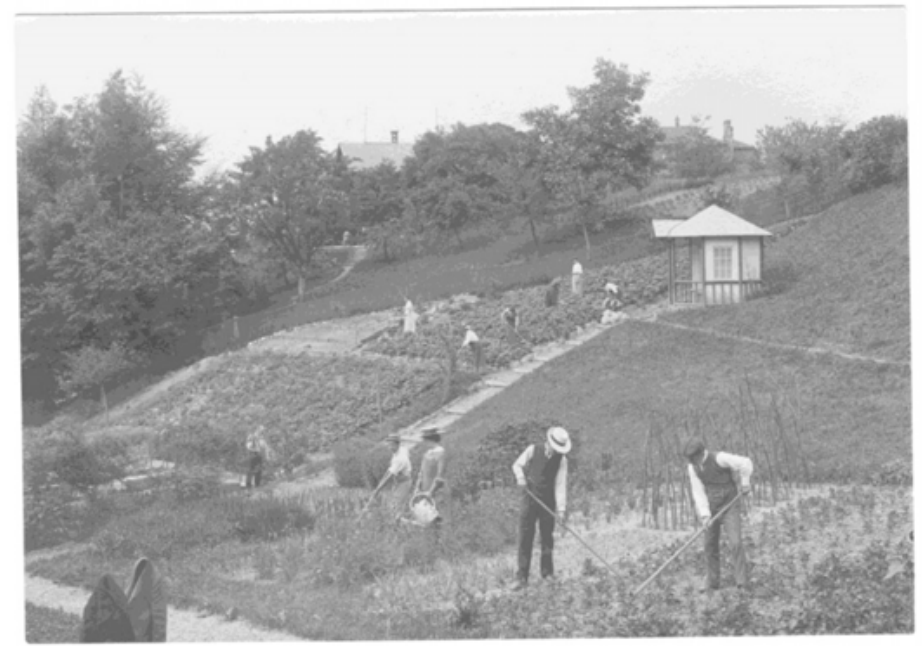

Abbildung 3: Gartenarbeit von Patienten auf dem Gelände des Sanatoriums um 1910 (Bircher-Benner-Archiv, Universität Zürich). 


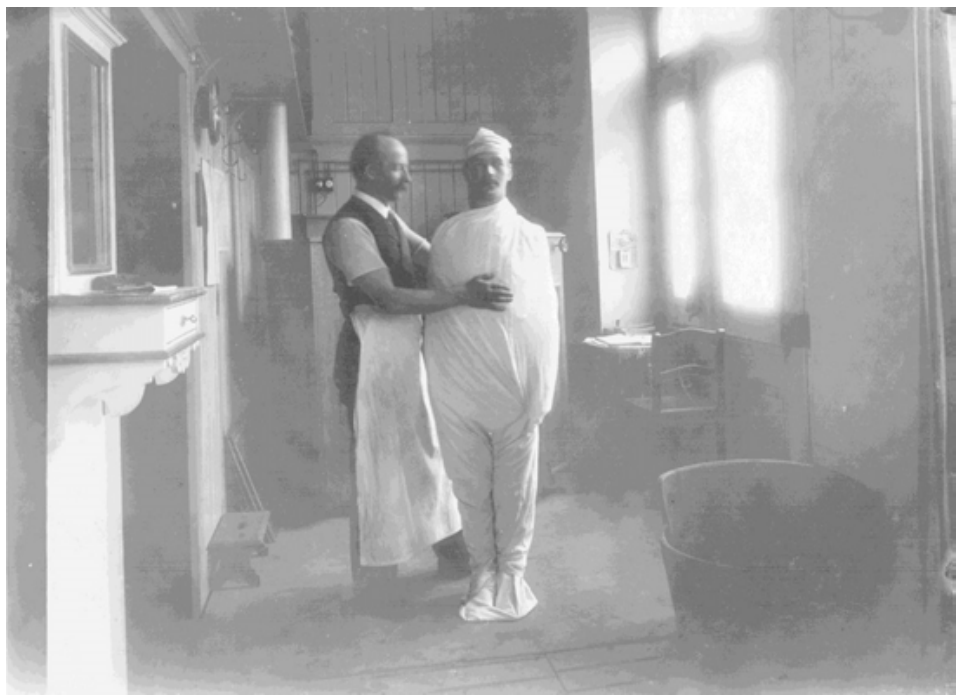

Abbildung 4: Naturheilkundliche Anwendung (Ganzkörperwickel) im Sanatorium um 1910 (Bircher-Benner-Archiv, Universität Zürich).

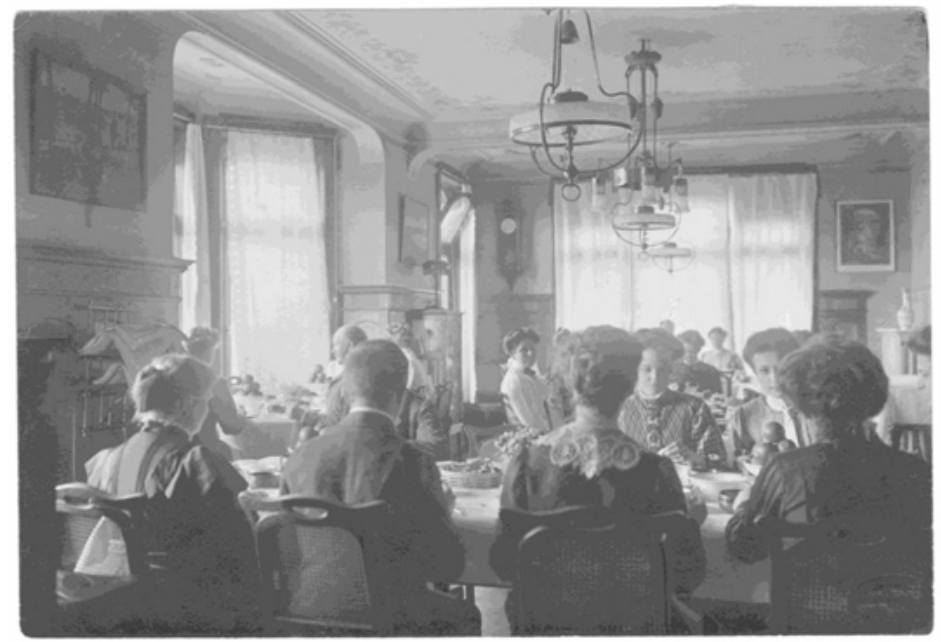

Abbildung 5: Der Speisesaal des Sanatoriums um 1910 (Bircher-BennerArchiv, Universität Zürich). 


\section{Landschaft als spezifische Anordnung einschlägiger Merkmale}

$\mathrm{Zu}$ einer kulturellen Landschaft wurde das Sanatoriumsgelände nicht allein durch die bloße Häufung gesundheitsrelevanter Merkmale. Wesentlich war zudem ihre Struktur. So waren viele der gesundheitlichen Orte wie auch Praktiken von innen nach außen, also in Richtung auf die zentralen naturheilkundlichen Elemente Licht, Luft und Sonne, gerichtet, nicht nur die diversen Gesundheitspraktiken wie der ritualisierte morgendliche Spaziergang. Die Hauszeitschrift "Der Wendepunkt im Leben und Leiden" machte die gesundheitliche Denkrichtung "aus dem Haus ins Freie“ über mehrere Jahre im Titelsignet explizit (Abb. 6).

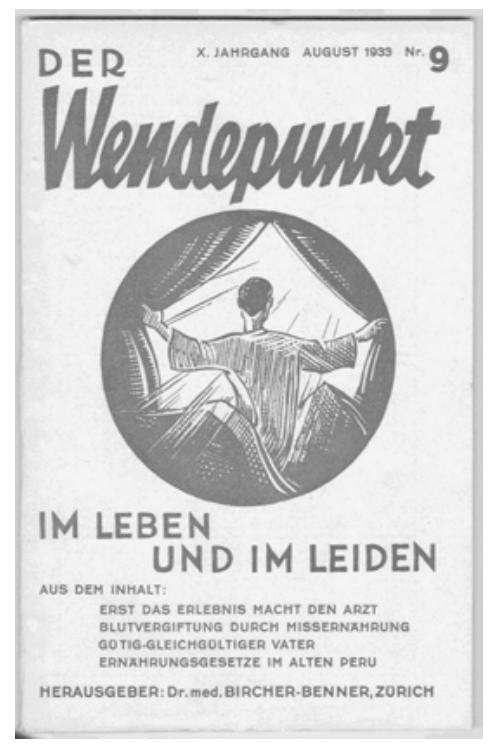

Abbildung 6: Titelblatt der Hauszeitschrift "Wendepunkt" (BircherBenner-Archiv, Universität Zürich).

Ein weiteres wesentliches Strukturmerkmal ist die Anordnung von Architekturstilen auf dem Klinikgelände. Dies erschließt sich allerdings erst auf den zweiten Blick. Während das ursprüngliche Haupthaus in dem für das gehobene städtische Randquartier des Zürichbergs typischen schweizerischen Heimatstil gehalten ist, 
sind die beiden ersten Folgebauten, zwei Gästehäuser, im alpinen "Chalet"-Stil gebaut (Abb. 7). Ein drittes „Chalet" sollte später hinzukommen, so dass sie zusammen eine stilistisch einheitliche Häuserflucht bildeten. Diese Anordnung ist ein Abbild der Lage des Sanatoriums innerhalb seiner Umgebung. Die Lage, also die räumliche Beziehung des Ortes zu anderen Orten, zeichnete sich nämlich ebenfalls durch Zweigleisigkeit aus.

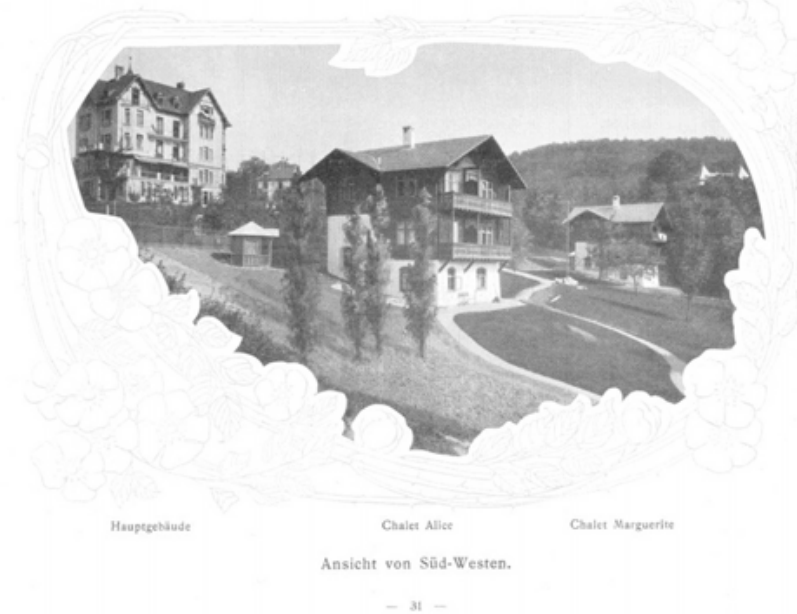

Abbildung 7: Das Haupthaus und die beiden Chalets des Sanatoriums. Aus einem Prospekt des Sanatoriums von 1907 (Bircher-Benner-Archiv, Universität Zürich).

Das Klinikgelände hatte eine doppelte Anbindung. Die erste war diejenige an die Stadt. Vom Haupthaus ging es auf die Straße und von dort in einem kurzen Fußweg zur Endhaltestelle der Straßenbahn, einem zu dieser Zeit eindeutigen Symbol moderner Stadtkultur und Mobilität (siehe Abb. 2). Die Anbindung der Klinik an das moderne Nahverkehrssystem war mehr als die Pragmatik, den Patientinnen und Patienten die Anreise leichter $\mathrm{zu}$ machen. Diese Anbindung war auch in konzeptueller Hinsicht wichtig. Das Sanatorium war nicht wie manche lebensreformerischen Kolonien als antizivilisatorischer Rückzug in die Natur gedacht, sondern als Nutzung natürlicher Heilkräfte in einem modernen Ambiente. Entsprechend waren die Patienten keine gesellschaftlichen 
Aussteiger, sondern bürgerliche, meist städtische Sanatoriumsgäste bei einer Kur, die sich nicht einfach von ihrem kulturellen Hintergrund trennen, sondern in ihm gesund und naturgemäß leben wollten. Die nahe Straßenbahn bedeutete eine Anbindung an die Stadt und damit gleichzeitig eine Bindung an die bürgerliche Lebensrealität der Patienten. Auch das Sanatorium „Lebendige Kraft" selbst bot die Möglichkeit zu normalem bürgerlichem Wohnen mit modernem Komfort. Bircher-Benner verstand seine "Ordnungstherapie" nicht als antizivilisatorischen Rückzug. So hatte er keine Probleme damit, dass die Heliotherapie mit elektrischen Lichtbädern praktiziert wurde.

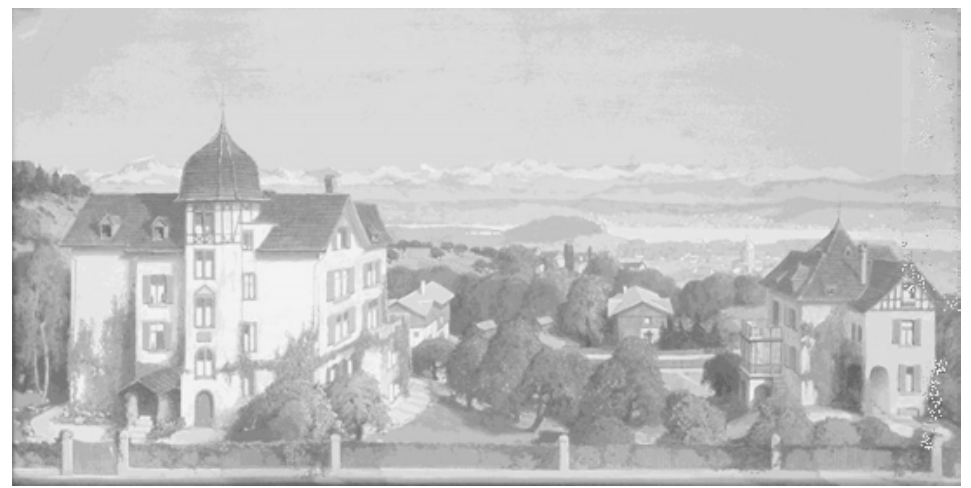

Abbildung 8: Ölgemälde eines nicht näher bekannten Patienten der Klinik (ca. 1910) (Bircher-Benner-Archiv, Universität Zürich).

Die zweite Anbindung des Geländes über seine Lage war optischer Art. Die Chalets lagen vom Haupthaus aus hangabwärts in Richtung See und Alpen. Die Sicht auf die Natur war ein wesentliches Merkmal der Lage des Sanatoriums. (Abb. 8). Der oft bewunderte Ausblick auf die typisch schweizerische Naturlandschaft war ein deutlicher Verweis auf die ursprüngliche Natur und ihre gesundheitliche Bedeutung. Zum einen lieferte die Natur Therapieelemente wie reine, frische Luft, Licht und Wasser. Zum anderen waren die Alpen für Bircher-Benner und viele Naturheiler der ideelle Ort der rückwärtsgewandten Utopie einer absoluten Gesundheit im Naturzustand. So kolportierte Bircher-Benner als Ursprungsmythos seines „Müesli“ die Bekanntschaft auf einer Bergwanderung mit einem Bergbauern, der sich vornehmlich mit 
Äpfeln, Milch und Getreide ernährte und gleichzeitig behauptete, in seinem Leben nie krank gewesen zu sein. ${ }^{3}$

Besonders wichtig ist, dass sich das Sanatorium nicht in den Bergen befand, sondern auf einem stadtnahen Hügel im Angesicht der Berge. Es ging um die Möglichkeit einer ästhetischen, sinnlichen Rezeption der „Urnatur". Das zu bestaunende Hochgebirge war für den Durchschnittsmenschen des frühen 20. Jahrhunderts auch nicht einfach zugänglich. In diesem Sinne handelt es sich nicht um einen real erreichbaren Ort, sondern einen Idealzustand von Natürlichkeit, eine Utopie im wörtlichen Sinne des Begriffes, einen "Nicht-Ort". Die Berge als unerreichbare Utopie von Natürlichkeit müssen fern bleiben. Das Hochgebirge hat hier eine ästhetische Funktion. Die Berge sind Staffage, Hintergrund einer Projektionsfläche für Empfindungen im Sinne der klassischen ästhetischen Naturlandschafts-Rezeption der Neuzeit (vgl. Fischer 2008: 22f). Der Architekturstil der Chalets ist ein Zitat dieser Vorstellung.

So repräsentierten einzelne Merkmale dieser speziellen medikalen Landschaft des Sanatoriums, seine Architektur und seine Lage, dessen doppelte medizinisch-kulturelle Ausrichtung. Das Sanatorium steht als räumliches Bindeglied zwischen dem zivilisierten Stadtleben sowie der emotionalen Naturüberhöhung und vereint beides in sich. In der Raumanordnung der medikalen Landschaft spiegelten sich so die Vorstellungen des Betreibers sowie der Gäste.

Eine ganz ähnliche doppelte Raumbeziehung konstruierte Bircher-Benner nochmals auf privater Ebene. Er wählte Braunwald im Kanton Glarus als Ort des Ferienhauses für sich und seine Familie. Braunwald ist von Zürich aus einer der nächstgelegenen Orte am Fuße des Hochgebirges. Bircher-Benner, ein Liebhaber großer und kräftiger Limousinen, konnte Braunwald per Straße oder Schiene leicht erreichen, indem er ans andere Ende des Zürichsees fuhr und dann durch das Tal der Linth, einem Zufluss des Zürichsees. Eine Standseilbahn (eröffnet 1907) brachte ihn schnell und bequem die letzten 600 Höhenmeter hinauf nach

3 Bircher-Benners Sohn Ralph verlegte mit seinem Buch über die „Hunsa. Das Volk, das keine Krankheit kennt", Bern 1942, den Ort der Gesundheitsutopie noch weiter weg, nämlich in den Hindukusch. 
Braunwald, wo er zwar nicht in, aber doch in nächster Nähe und im Angesicht des Hochgebirges war. In einem Foto ist dieses Verhältnis zur Natur paradigmatisch festgehalten. (Abb. 9). Es zeigt Bircher-Benner im Garten seines Ferienhauses inmitten der Natur, einem lichten Gehölz, selbst aber mit den zivilisatorischen Insignien eines weißen Anzuges, weißer Schuhe, eines eigens aufgestellten Tisches und Stuhls sowie einer Schreibmaschine ausgestattet.

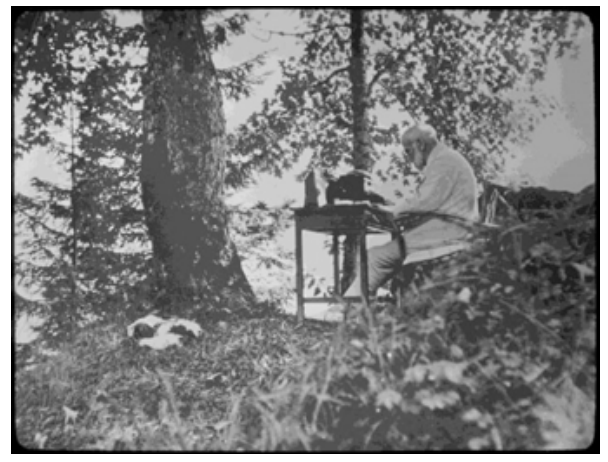

Abbildung 9: Max Bircher-Benner vor seinem Ferienhaus in Braunwald (undatiert) (Bircher-Benner-Archiv, Universität Zürich).

\section{Landschaft als Einheit}

Noch in einem weiteren Sinn konstituiert sich das Sanatorium durch die Struktur bestimmter Merkmale als kulturelle Landschaft. Spätestens mit dem Zubau eines repräsentativen „Privathauses", bildeten die Gebäude zusammen ein Ensemble, der eine Art Mikrokosmos der gesunden Lebensweise und Selbstsorge (vgl. Wolff 2010c) um den eigenen Körper wurde. Schon früher kommt diese Idee des Mikrokosmos in einer vedoutenähnlichen Darstellung des Klinikgeländes zum Ausdruck, die in einem Sanatoriumsprospekt abgebildet wurde (Abb. 10). Das Sanatorium wird darin idealisiert als ein eigener Gesundheitskosmos abgebildet, indem die Nachbarhäuser in den Hintergrund gerückt sind und stattdessen die in Wirklichkeit entferntere Naturlandschaft das Sanatorium direkt umgibt, ja einbettet. Die Übergänge von der Naturlandschaft zur Sanatoriumslandschaft sind fließend. Das eigentliche Sanatoriumsgelände ist gegenüber der Realität extrem vergrößert. Es vereint Spazierwege, Parkanlagen, Lufthütten, ein 
Luftbad, Beete und einen Turnplatz. Die Abbildung erinnert an einen Landschaftsgarten, der ja selbst eine gesamte Lebenswelt (und nicht nur eine Naturlandschaft) in idealisierter Form abbilden soll. Gehen wir davon aus, dass ein Landschaftsgarten in seiner Konstruiertheit ein Paradebeispiel für eine kulturelle Landschaft darstellt, findet sich in dieser Darstellung die Idee des Sanatoriums als medikaler Landschaft am deutlichsten repräsentiert.

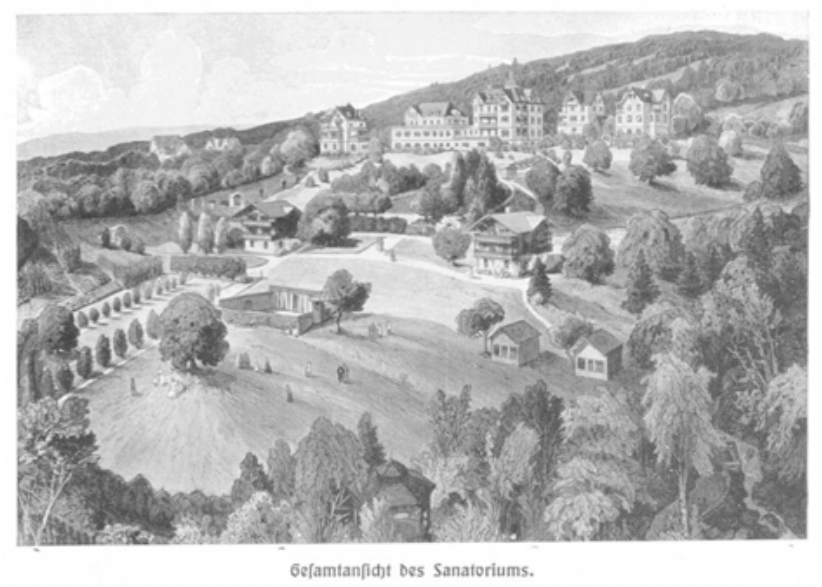

Abbildung 10: Darstellung des Klinikgeländes aus einem Sanatoriumsprospekt des Jahres 1913 (Bircher-Benner-Archiv, Universität Zürich).

\section{Landschaft als Produkt von Wahrnehmung}

Landschaft im kulturwissenschaftlichen Sinn existiert nicht aus sich selbst heraus. Zum einen ist sie, wie gezeigt, konkret gemacht. Zum anderen entsteht eine spezifische Landschaft erst durch eine bestimmte Wahrnehmungsweise dieses Ausschnittes der Umwelt, ähnlich wie eine Naturlandschaft nicht per se romantisch und eine Stadtlandschaft erst durch eine spezifische Wahrnehmung und Bewertung zur "Häuserwüste“ wird. In diesem Sinne könnte man die zuletzt genannte Darstellung des Sanatoriums als Landschaftsgarten nicht nur als einen propagandistischen Trick für einen Werbeprospekt interpretieren, sondern darin auch sozusagen eine „Mental Map“ entdecken, auf der nicht Wirklich- 
keit abgebildet ist, sondern eine Art der Empfindung dieser Wirklichkeit. Wie und warum auch immer diese Abbildung entstanden sein mag, zielt sie doch auf eine spezifische Vorstellung von diesem Sanatorium, nämlich der als Landschaftsgarten, als eingebettet in die Naturlandschaft, als idealer Ort, als Ort des Wohlergehens und der Gesundung etc.

Das bereits erwähnte Ölgemälde eines unbekannten Patienten (Abb. 8) zeigt diesen Umstand ebenfalls. Die Perspektive mit dem Hauptgebäude im Vordergrund und Stadt, See und Alpen im Hintergrund ist nicht die naheliegendste, weil sie von einem Punkt außerhalb des Sanatoriums auf der anderen Seite der Straße ausgeht. Die auf dem Gemälde erscheinende enge Verbindung zwischen den Hauptgebäuden, den Chalets und der Naturlandschaft ist also mehr oder weniger bewusst hergestellt, und es ist anzunehmen, dass diese Einheit so von der malenden Person auch wahrgenommen wurde. Auf Sanatoriumsprospekten dominierte lange eine entgegengesetzte Perspektive auf die Frontseite der Gebäude (z.B. Abb. 7). Fotos mit See und Alpen tauchen in der Eigenwerbung erst in den 1980er Jahren auf.

\section{Die Landschaft als symbolische Repräsentation einer Idee durch die Aufladung mit "Sinn“}

Zur kulturellen Landschaft wird ein Raum letztlich erst dadurch, dass ihm aus dieser spezifischen Wahrnehmung auch eine spezifische Bedeutung, ein Sinn zugesprochen wird, wie es in den definitorischen Passagen zu Beginn bereits angesprochen worden war. Die medikale Landschaft des Zürcher Sanatoriums war in diesem Sinne hochgradig mit gesundheitsbezogenem "Sinn" aufgeladen. Das Sanatorium wurde so Identifikations- und Repräsentationsort einer spezifischen Idee von Gesundheit durch Naturnähe.

\section{Mehrdeutigkeiten kultureller Landschaften}

Dass eine Bedeutungszuweisung der medikalen Landschaft in dieser Form nicht zwangsläufig ist, zeigen alternative Sinngebungen. Für Thomas Mann, im Jahre 1909 selbst Patient des Sana- 
toriums, war die Naturnähe eher fremd, er fühlte sich dort zum "Gras essenden Nebukadnezar" gemacht, „der im Luftbade auf allen Vieren geht". Die Bedeutung des Sanatoriums lag für ihn eher - und typisch für ihn selbst - in der intensiven Selbstsorge um den eigenen Körper sowie seiner Selbstüberwindung zu einem Leben im Rahmen der Ordnungstherapie. Mann bezeichnete das Sanatorium aufgrund des strengen Tagesregimes als „hygienisches Zuchthaus". Es sei ein Ort,

„wo man um 6 Uhr aufstehen, um 9 Uhr das Licht löschen muss und den Tag unter Luft- und Sonnenbädern, Wasseranwendungen und Gartenarbeit verbringt. Das ist hart, zu Anfang und während der ersten fünf Tage stand ich beständig mit trotzigen Entschlüssen ringend vor meinem Koffer (...). Aber obgleich ich mehr für Voltaire als für JeanJacques (Rousseau, E.W.) bin, bereue ich es doch gar nicht, durchgehalten $\mathrm{zu}$ haben. Meine störrische Verdauung besserte sich dann ins Erstaunliche, Niedagewesene." (Virchow 2002: 191)

\section{Relationale Konstituierung von Landschaft}

Räume können ihren Charakter als kulturelle Landschaften auch dadurch erhalten, dass sie sich von Räumen mit gegenteiligen Merkmalen, sozusagen "Gegen-Landschaften“, absetzen und damit deutlicher hervortreten. Im Falle des Sanatoriums von BircherBenner war etwa das nahe gelegene Hotel Dolder partiell eine solche Gegenlandschaft. Was die rigide Lebensführung und vor allem den Speisezettel betraf, war das Luxushotel das blanke Gegenteil und aus rohköstlerischer Sicht die Inkarnation einer Ungesundheits-Landschaft. Gerade kontrastiv wird es somit zum konstitutiven Teil der medikalen Landschaft von Bircher-Benners Sanatorium.

\section{Landschaften als unscharfe Räume}

Das mögliche Gegenüber von Landschaft und Gegenlandschaft darf nicht zu der Annahme verleiten, dass (medikale) Landschaften grundsätzlich scharfe Grenzen haben müssen. Im Gegenteil, sie können Zonen des fließenden Übergangs zu ihrer Umgebung 
haben. Auch hier bietet das Sanatorium „Lebendige Kraft” ein anschauliches Beispiel. Seine Absetzung von der Umwelt war nicht absolut. Es dürfte nur teilweise durch einen Zaun von den umliegenden Grundstücken getrennt gewesen sein, vor allem ging es an einer Seite fließend in den Tobel des Wolfbachs über (Abb. 7), also einen viel deutlicheren Naturraum. Der obligate Morgenspaziergang der Patienten bedeutete zudem, dass diese die im Sanatorium repräsentierte Lebensweise täglich auf die Umgebung ausdehnten. Dass es hier zum Aufeinandertreffen unterschiedlicher Gesundheitskulturen (und mithin zur Überschneidung medikaler Landschaften) kommen konnte, ist in der Karikatur eines Patienten aus den 1970er Jahren festgehalten (Abb. 11).

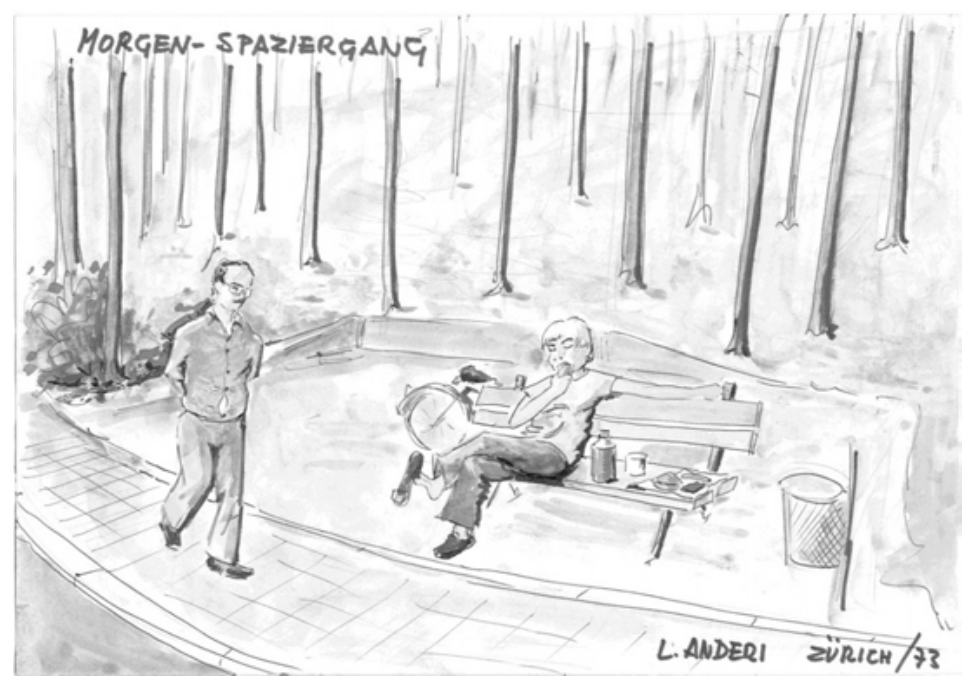

Abbildung 11: "Morgen-Spaziergang“. Karikatur eines Klinikgastes von 1973 (Bircher-Benner-Archiv, Universität Zürich).

\section{Landschaften als Netzwerke}

Das Rohkost-Sanatorium Bircher-Benners war kein singuläres Phänomen, selbst in seiner direkten Umgebung. In der Nähe fanden sich zumindest teilweise ähnlich strukturierte GesundheitsOrte wie das bereits eingeführte Grand Hotel Dolder, das sich, ganz ähnlich dem Bircher-Sanatorium, als gesundheitlich wertvoller „Höhenkurort" und touristisches Ziel mit See- und Alpenblick 
verkaufte. Mehrere Mikro-Landschaften konnten und können sich somit als Netzwerke zu Makro-Landschaften formieren (Hoyez 2007), z.B. zur größeren Zürcher oder schweizerischen Sanatoriums- bzw. Kurort- oder gar Gesundheits-Landschaft (Graf/Wolff 2010). Unterschiedliche Landschaftsarten können sich damit auf verschiedenen Ebenen konstituieren. Oder sie überschneiden sich mit anderen, z.B. Tourismuslandschaften.

\section{Landschaften mit historischer Dynamik als "sedimentierte Geschichte“}

Es darf dabei auch nicht übersehen werden, dass Landschaften nicht per se stabil sind, sondern einem historischen Wandel unterliegen. Das Ensemble des Sanatoriums „Lebendige Kraft“ wuchs nicht nur. Es veränderte auch seine Funktion ein Stück weit. In den Jahrzehnten nach dem Zweiten Weltkrieg wandelte sich der Name in „Privatklinik Bircher-Benner", die Einrichtung wurde so ein Stück weit vom Sanatorium zur Klinik, in der zunehmend unterschiedliche alternative Heilweisen angeboten und eher kranke Patienten therapiert wurden als dass Menschen mit angeschlagener Gesundheit hier die Ordnungstherapie einübten. 1994 schließlich wurde die Klinik geschlossen, verkauft, umgebaut und 2000 als Tagungszentrum eines Finanzdienstleisters wiedereröffnet. ${ }^{4}$ Die abstrakte (medikale) Landschaft änderte sich damit inhaltlich, einmal graduell, einmal grundlegend. Dies schlug sich teils auch in der konkreten Landschaft wider. Ein Therapietrakt wurde gebaut, die Lufthütten und Gemüsebeete verschwanden. Gleichzeitig verblieben alte Elemente auch in der veränderten Landschaft. Wer das Gelände heute betrachtet, sieht diese Elemente wiederum in veränderter Funktion. Der Therapietrakt beherbergt heute Tagungsräume. Gleichwohl scheint aus ihnen zum Teil noch die alte Funktion hervor. Aktuelle konkrete Landschaften können somit auch „sedimentierte Geschichte“ darstellen.

4 S. www.zurichdevelopmentcenter.com. 


\section{Fazit}

Die vorangegangenen Überlegungen sollten zeigen, wie sich Ideen in konkreten Räumen abbilden und dass sich die spezifischen Erscheinungsformen dieser Räume zu einer Struktur formieren können, die einen gewissen inneren Zusammenhang haben, welche den Raum zu einer kulturellen Landschaft machen. Im gegebenen Beispiel heißt das, dass sich die spezifische Vorstellungswelt eines Sanatoriums von gesunder Lebensführung auch räumlich abbildet, was das Gelände zu einer "medikalen Landschaft" werden lässt. Umgekehrt formuliert kann die konkrete Form dieser Landschaft Hinweise auf die hinter ihr stehenden Vorstellungen und Ideen geben. Ein weiter kulturwissenschaftlicher, relativ abstrakter Landschaftsbegriff, der sich von der engen Verbindung mit Naturlandschaften löst, hat auf diese Weise ein interessantes heuristisches Potenzial.

Andere Aspekte einer Raumanalyse blendet der Landschaftsbegriff allerdings eher aus. So ließen sich z.B. auch Wege bzw. Routen fruchtbar analysieren, etwa diejenigen der Patienten zum, im und vom Sanatorium als "transitorischem Raum", wobei Zusammenhänge zwischen der symbolischen Ortsveränderung der Kur und der angestrebten gesundheitlichen Verhaltensänderung untersucht werden könnten.

Wenn mit dem vorgeschlagenen Instrumentarium einzelne Landschaften herausgearbeitet werden können, bedeutet dies nicht, dass hier einem essentialistischen Landschaftsbegriff einer singulären, abgeschlossenen, eindeutigen, konstanten Landschaft das Wort geredet wird. Die Methode soll keinesfalls über die vielen gegenwärtigen Prozesse der Veränderungen von Raumstrukturen hinweggehen, die unter dem Begriff der „Verflüssigung“ von Räumen zusammengefasst werden. Im Gegenteil: Kulturelle Landschaften, auch medikale, können immer als vieldeutige, veränderliche, unscharfe und plurale oder auch zunehmend virtualisierte Phänomene betrachtet werden. Auch dies bedarf eines analytischen Instrumentariums. 


\section{Literatur}

Ackerknecht, Erwin H. (1963): Geschichte und Geographie der wichtigsten Krankheiten. Stuttgart: Enke.

Fischer, Norbert (2008): Landschaft als kulturwissenschaftliche Kategorie. In: Zeitschrift für Volkskunde 104, S. 19-31.

Gesler, Wilbert M. (1992): Therapeutic landscapes: medical issues in light of new cultural geography, in: Social Science and Medicine 34, S. 735-746.

Gesler, Wilbert M. (2005): Editorial: Therapeutic landscapes: An evolving theme, in: Health and Place 11, S. 295-297.

Graf, Felix und Wolff, Eberhard (Hg.) (2010): Zauber Berge. Die Schweiz als Kraftraum und Sanatorium, Baden: hier und jetzt (im Druck).

Hoey, Brian H. (2007): Therapeutic uses of place in the intentional space of purposive community. In: Williams, Allison (Hg.): Therapeutic landscapes. Aldershot: Ashgate, S. 297-314.

Hoyez, Anne-Cécile (2007): From Rishikesh to Yogaville: the globalization of therapeutic landscapes, in: Williams, Allison (Hg.): Therapeutic landscapes. Aldershot: Ashgate, S. 49-64.

Kaufmann, Stefan (2005): Soziologie der Landschaft. Wiesbaden: VS.

Löw, Martina (2001): Raumsoziologie. Frankfurt am M.: Suhrkamp.

Seifert, Manfred/Krebs, Stefanie u.a. (Hg.) (2010): Landschaft quer

Denken. Theorien - Bilder - Formationen. Leipzig: Universitätsverlag (im Druck).

Virchow, Christian (2002): Das Sanatorium als Lebensform. Über einschlägige Erfahrungen Thomas Manns, in: Sprecher, Thomas (Hg.): Literatur und Krankheit im Fin-de-siècle (18901914). Thomas Mann im europäischen Kontext. Frankfurt am M.: Vittorio Klostermann, S. 171-197.

Williams, Allison (Hg.) (1999): Therapeutic landscapes. The dynamic between place and wellness. Lanham: Univ. Press of America.

Williams, Allison (Hg.) (2007a): Therapeutic landscapes. Aldershot: Ashgate.

Williams, Allison (2007b): Introduction: The continuing maturation of the therapeutic landscape concept, in: Dies. (Hg.): Therapeutic landscapes. Aldershot: Ashgate, S. 1-12. 
Wolff, Eberhard (Hg.) (2010a): „Lebendige Kraft". Max BircherBenner und sein Sanatorium im historischen Kontext, Baden: hier und jetzt (im Druck).

Wolff, Eberhard (2010b): Das Phänomen Bircher-Benner. In: Ders. (Hg.): „Lebendige Kraft“. Max Bircher-Benner und sein Sanatorium im historischen Kontext, Baden: hier und jetzt (im Druck).

Wolff, Eberhard (2010c): Moderne Diätetik als präventive Selbsttechnologie: Zum Verhältnis heteronomer und autonomer Selbstdisziplinierung zwischen Lebensreformbewegung und heutigem Gesundheitsboom, in: Lengwiler, Martin/Madarasz, Jeannette (Hg.): Transformationen des präventiven Selbst. Vorsorgepraktiken in der Moderne, Bielefeld: transcript (im Druck). 
Bereitgestellt von | Universitaetsbibliothek Basel

Angemeldet

Heruntergeladen am | 18.09.18 17:45 